\title{
BMJ Open Doctors' engagement with a formal system of continuing professional development in Ireland: a qualitative study in perceived benefits, barriers and potential improvements
}

\author{
Holly Rose Hanlon (D , ${ }^{1}$ Lucia Prihodova, ${ }^{1}$ Thelma Russell, ${ }^{2}$ Deirdre Donegan, ${ }^{2}$ \\ Ann O'Shaughnessy, ${ }^{3}$ Hilary Hoey ${ }^{2}$
}

To cite: Hanlon HR,

Prihodova L, Russell T, et al. Doctors' engagement with a formal system of continuing professional development in Ireland: a qualitative study in perceived benefits, barriers and potential improvements. BMJ Open 2021;11:e049204. doi:10.1136/ bmjopen-2021-049204

- Prepublication history for this paper is available online To view these files, please visit the journal online (http://dx.doi org/10.1136/bmjopen-2021049204).

Received 04 May 2021 Accepted 18 0ctober 2021

Check for updates

(c) Author(s) (or their employer(s)) 2021. Re-use permitted under CC BY-NC. No commercial re-use. See rights and permissions. Published by BMJ.

${ }^{1}$ Research Department, Royal College of Physicians of Ireland, Dublin, Ireland

${ }^{2}$ Professional Competence Department, Royal College of Physicians of Ireland, Dublin, Ireland

${ }^{3}$ Professional Affairs, Royal College of Physicians of Ireland, Dublin, Ireland

Correspondence to Holly Rose Hanlon;

HollyHanlon@rcpi.ie

\section{ABSTRACT}

Objectives To examine experiences of participation in a mandatory system of continuing professional development (CPD) among doctors in Ireland, in order to identify areas for improvement.

Design A qualitative cross-sectional design was used. Participants 1408 participants (701 male, 707 female) were recruited via email from a population of 4350 doctors enrolled on a Royal College of Physicians of Ireland Professional Competence Scheme (PCS) for the 2017/2018 year, and completed an online survey as part of a larger study examining experiences and attitudes towards participation in PCS. A subset of the sample (434 participants) responded to an optional open-ended question about PCS participation. Responses to the openended question were analysed using thematic analysis.

Results Thematic analysis resulted in five main themes relating to perceived barriers to PCS participation across a wide range of areas: 'Evidence of participation', 'The structure of PCS', 'Questioning the benefits of formal CPD', 'Workplace challenges' and 'Access issues'.

Conclusions Taken together, the five themes outlined in this study give a wide-ranging, in-depth picture of the challenges faced by Irish doctors, which expand on welldocumented factors such as time constraints, to illustrate a series of complex, interacting factors. Some barriers, such as difficulty obtaining evidence of participation, may be relatively easily addressed. Others, such as issues with the way the PCS is structured, are more intractable, and require further research to understand more fully and develop appropriate solutions.

\section{BACKGROUND}

Continuing professional development (CPD) in medicine, while long considered an important part of the profession, is increasingly becoming mandatory, and is often linked to revalidation or recertification procedures for doctors. ${ }^{12}$ CPD generally refers to the broad range of skills and knowledge needed to effectively practice medicine, including general skills such as management,

\section{Strengths and limitations of this study}

- The present study describes novel barriers to engagement with a formal system of mandatory continuing professional development (CPD), with the qualitative nature of the study allowing for a more in-depth examination of the issues outlined by participants (eg, the structure of Professional Competence Scheme, PCS) which have not been examined in previous work, at a level of detail that is often missed in quantitative studies.

- This study is limited by its use of a single openended anonymous question, with no opportunity for follow-up questions. However, future research is planned to expand on the findings of this study in more depth.

- In addition, it must be noted that a majority of participants did not respond to this optional question, and the results of the larger study suggest that the majority of doctors are satisfied with the PCS system and believe it is beneficial and improves patient care and safety. ${ }^{20}$

- It may, therefore, be the case that responses analysed in this study disproportionately represented those with more complaints about formal CPD. However, this group - those who perceive and experience significant barriers to PCS participationare the group who are least likely to meet their CPD requirements, and therefore, their needs must be identified in order to design interventions aimed at improving their performance.

teaching and leadership skills as well as specific medical knowledge; in contrast the term 'continuing medical education' generally refers to medicine-specific knowledge and skills.

Multiple studies have shown CPD to be effective at improving doctor performance and patient outcomes, although this has been found to vary across different CPD 
activities, with more interactive CPD activities using multiple learning methods typically found to have more positive outcomes than other CPD activities, such as lectures. ${ }^{3}{ }^{4}$ However, many doctors must navigate numerous barriers to participate fully and successfully in CPD. While doctors generally accept the need for participation in lifelong medical learning throughout their career, the introduction of formal, mandatory CPD can represent an additional pressure for already overworked doctors. ${ }^{56}$ Therefore, there is a need to determine the barriers and facilitators to successful CPD engagement, in order to maximise outcomes for both doctors and patients.

\section{PREVIOUSLY IDENTIFIED BARRIERS AND FACILITATORS TO PARTICIPATION}

The most frequently reported barriers to CPD participation in the research literature are time, cost and location of CPD activities, likely reflecting the high-pressure, timeconstrained nature of medical practice. For example, recent study by Maher $e t a t^{6}$ found all three factors to be significant barriers to hospital doctors in Ireland. This pattern has been reflected in the international literature; in a survey of Australasian geriatricians; Etherton-Beer et $a l^{7}$ also found time constraints (both regarding professional and non-professional commitments) to be a major barrier, however, this study did not find cost to be a major barrier, potentially reflecting differences in remuneration for doctors across countries. Similar results were found by Weiland $e t a l^{8}$ and a second study by Dent $e t a l,{ }^{9}$ which also expanded on these results with qualitative items which indicated that fatigue, staff shortages, valuing family time and a lack of relevant, good quality CPD activities for doctors were also prominent barriers. This pattern of lack of relevant CPD activities, time constraints and the financial cost of CPD activities as being significant barriers to engagement is repeated in numerous studies. ${ }^{1011}$

Along with the shift towards mandatory CPD, more negative perceptions and attitudes towards CPD have been found among some doctors, which may contribute to a lack of enthusiasm for participation in CPD activities. For example, Macdougall et $a l^{12}$ outlined the pros and cons of compulsory CPD versus unregulated, self-directed learning, noting the feeling that mandatory CPD may turn into a 'tick-box' exercise with more focus on requirements than on whether learning is actually taking place. They also note that reflection is often a requirement of CPD schemes, but question whether 'reflection by rote' as part of accreditation is as valuable as the kind of reflection that arises spontaneously as part of everyday practice. ${ }^{12}$ After the recent implementation of a CPD system that is integrated into the process of medical revalidation in the National Health Service (NHS), while noting that they considered the appraisal process to be valuable, UK doctors expressed fears that by integrating the appraisal process (including CPD, reflection and audit) into revalidation, it could become a bureaucratic tick-box exercise. ${ }^{13}$
This perception that CPD is more 'for show' than of any real value for patient outcomes has been found more explicitly in a study of paediatricians in the USA, which found that under half of participants agreed that participating in mandatory CPD activities helped them to meet their lifelong learning goals, while only 1 in 10 agreed that participation helped their patient care. ${ }^{11}$ These negative perceptions toward CPD are notable, as negative attitudes have been found to impact motivation and engagement with activities, especially when the activity is seen as burdensome. For example, Deitrich $e t$ $a l^{14}$ found that tasks with a low perceived value and a high perceived cost are associated with less motivation and effort. It may be the case that if doctors see mandatory CPD as an expensive, time consuming burden with little benefit, then this may act as a barrier to their participation.

However, these perceptions are at odds with the research literature which show that high-quality CPD activities can be effective at improving both doctor and patient outcomes, with CPD activities that are more interactive and which involve multiple learning methods generally found to be more effective than either didactic lectures or online courses. ${ }^{3415}$ The results of the above studies indicate a need to emphasise to doctors the importance of CPD in the rapidly changing world of medicine and also the need for access to high-quality relevant learning activities.

\section{CPD IN IRELAND}

One country which has made the change to a formal system of CPD in the last decade is Ireland. In the Irish medical system, since 2011, all doctors are required to enrol on a Professional Competence Scheme (PCS). Doctors in Ireland must complete 50 hours total of CPD per year, along with a minimum of one audit or quality improvement activity. ${ }^{16}$ Doctors choose their own CPD activities based on their needs and scope of practice within an adaptable framework. A minimum of 20 credits must be recorded in the internal category (practice evaluation and development), 20 in external (maintenance of knowledge and skills), 5 in personal learning and at least 5 more in a category of their choice, including the research and teaching category, which is optional. In addition, at least one credit must be recorded across each of the eight domains of good professional practice over a 5-year period, with the domains spanning areas from patient care and clinical skills to professionalism and collaboration. ${ }^{1617}$

The types of CPD activities for which doctors can claim CPD credits range from attendance at international conferences to journal clubs, clinical case presentations, review groups, team meetings, expert lectures, online learning and specialised training in both clinical and non-clinical skills, such as leadership, management and communication. ${ }^{6} 18$ 


\section{THE PRESENT STUDY}

While there has been an increase in research into CPD participation in recent years, the vast majority of studies focus on USA, Canadian or Australian samples. Few studies have examined perceptions of CPD participation in the context of the Irish health system, which has faced severe challenges, precipitated in part by the global economic recession, with budget cuts and staff shortages across the health service resulting in a stressful working environment for many doctors. ${ }^{19}$ This crisis has coincided with the introduction of mandatory CPD, highlighting the need for a focus on the perceived benefits and barriers to CPD among doctors in Ireland.

In addition, almost all the studies discussed above exclusively used forced-choice quantitative methods, in which doctors rated or ranked the significance of prechosen barriers. Such studies may fail to capture the full breadth of potential benefits and challenges experienced by doctors, and lack the depth necessary to fully understand the complex and often interacting factors which influence doctors' participation in CPD.

Therefore, a qualitative study investigating doctors' perceptions of the Royal College of Physicians of Ireland (RCPI) PCS was carried out as part of a larger survey-based study into doctors' future learning needs, and experiences and attitudes toward PCS. The study included participants working in both hospital and nonhospital-based settings, in order to explore the full spectrum of doctors' experiences in Ireland. The quantitative results of that study showed that over $80 \%$ of participants report that participation in PCS was a good idea, with the majority reporting positive experiences engaging with the process, and indicating that they felt PCS helped to improve their practice across a wide range of practice areas. ${ }^{20} \mathrm{~A}$ minority of participants reported experiencing difficulties, including a lack of time to participate and difficulties obtaining evidence of their participation in activities. ${ }^{20}$ The present study concerns the qualitative component of the survey measure. It was hoped that the open-ended nature of the question would allow for greater understanding of benefits, barriers and potential improvements to PCS in Ireland.

\section{METHOD}

\section{Participants}

A total of 1408 participants (701 male, 707 female) were recruited via email from a population of 4350 doctors enrolled on an RCPI PCS, indicating a response rate of $32 \%$.

\section{Patient and public involvement}

No patient involved.

\section{Design}

The study employed a cross-sectional design, with data collected in the form of a survey using both open-ended and Likert-scale questions, examining experiences and perceived benefits of participation in mandatory PCS. Details of the wider survey measure can be found elsewhere. ${ }^{20}$ For the purposes of this qualitative paper, analysis was limited to responses to the open-ended question 'Please include any additional comments you would like to provide about the benefits, barriers, or potential improvements that could be made to PCS'. A total of 434 doctors (or $30 \%$ of survey participants) responded to this question.

\section{Procedure}

RCPI operates 11 PCS across Ireland. All doctors who were enrolled on an RCPI PCS for the 2017/2018 year were invited via email to complete the online survey. Two additional reminder emails were sent after the initial email, spaced 3 weeks apart. Prior to participation, participants were required to read an online information sheet, which outlined the purpose of the study, and which stated that completion of the survey indicated consent. Participants then proceeded to the online survey at SurveyMonkey. com. Participants were eligible for CPD credit for their participation in the study.

\section{Analysis}

The anonymous raw data from the survey were downloaded from Survey Monkey in excel format. Responses to the open-ended question were analysed using thematic analysis as per Braun and Clarke. ${ }^{21} \mathrm{~A}$ total of 434 individual responses were first read in their entirety in order to familiarise the researcher with the data. Responses were then individually coded manually, resulting in 36 initial codes. Coded data extracts were collated, reread and refined, resulting in the collapse of a number of codes, into a final number of 30 . Individual codes were then reviewed and grouped into 13 initial themes.

These 13 themes were then reviewed for internal homogeneity and external heterogeneity ${ }^{21}$ and refined further.

\section{RESULTS}

The thematic analysis resulted in five main themes relating to attitudes and perceptions toward participating in CPD activities, with themes 1 and 2 each divided into two subthemes (see table 1 below). While the question was broad, and allowed for discussion of positive experiences of PCS participation, all five major themes found related to perceived barriers and areas for potential improvement.

\section{THEME 1: EVIDENCE OF CPD PARTICIPATION}

The first major theme in the data relates to the evidence of participation in CPD activities. Two distinct subthemes were found within this theme.

\section{Difficulties obtaining evidence of participation}

The first subtheme, 'difficulties obtaining evidence of participation' refers to the perception that one of the biggest issues in meeting the required amount of CPD 


\begin{tabular}{|c|c|}
\hline Theme & Subtheme \\
\hline Evidence of participation & $\begin{array}{l}\text { Difficulties obtaining evidence } \\
\text { of participation } \\
\text { Recording evidence of } \\
\text { participation }\end{array}$ \\
\hline The structure of PCS & $\begin{array}{l}\text { Issues with content of CPD } \\
\text { categories } \\
\text { PCS is inflexible and } \\
\text { unrealistic }\end{array}$ \\
\hline $\begin{array}{l}\text { Benefits of PCS versus } \\
\text { criticism of formal CPD }\end{array}$ & - \\
\hline Workplace challenges & - \\
\hline Access issues & - \\
\hline
\end{tabular}

CPD, continuing professional development; PCS, Professional Competence Scheme.

activities is in fact, proving participation. Doctors mention that there is a lack of a practical method of recording attendance on the part of CPD providers at the point of entry to activities. As one doctor states:

I don't really have evidence of attendance at many internal education meetings because it's just a sign in sheet and I don't know where it goes afterwards.

Additionally, it is often a difficult process to obtain a proof after CPD activities, and frequently the task of securing this proof falls on the doctors rather than the CPD providers. In one example, a doctor notes that the organiser of one CPD activity 'does not update the attendances', and that doctors 'have to email a few times just to update attendance and get certificates'. This difficulty may be more pronounced for certain types of CPD activity, particularly internal CPD which is less formal, and often takes place as part of the normal working day; 'Obtaining evidence of internal CPD (MDT (multidisciplinary team) meetings, etc) can be onerous' as there is 'no dedicated resource to collate attendance (need to photocopy individual attendance sheets, etc)'.

As one doctor puts it, 'The retention of data and the recording of participation is probably the biggest issue: lack of evidence is not the same as evidence of lack.' It appears that a significant barrier to doctors meeting requirements may be easily fixed by improving the ease at which doctors can obtain proof of attendance.

Doctors also express frustration at the fact that many of the learning or training activities they engage in are not formally accredited for CPD points, despite contributing to their professional development. This is particularly evident for those working in non-clinical specialties; 'As a doctor working in the pharmaceutical industry, I have recently noticed that it is very difficult to attain CPD certificates of attendance for the external meetings that I attend'.

Participants also note that some conferences, including more general science courses, may not have formal CPD accreditation as 'not enough doctors attend to make it worth their while.' Participants also cite the lack of CPD credits available for the time and effort involved in preparing for and taking medical exams; 'We prepare for exams, and we study for same. It also improves our knowledge a lot but it doesn't come under any category which is unfair'.

\section{Recording evidence of participation}

The second subtheme 'recording evidence of participation' refers to the process of actually recording CPD participation, namely challenges with using the online ePortfolio system used in Ireland. In particular, many doctors were of the opinion that the existing online system was 'clunky' and 'cumbersome'. Participants indicate that the process of inputting credits on the ePortfolio was arduous ('having to $\log$ in, fill in details and subsequently scan in or download certs and then upload, very cumbersome and time-consuming method of recording information') and that the prospect of having to engage with it could discourage doctors from recording their CPD credits in a timely manner:

I have got used to the online ePortfolio but I am conscious that some of my colleagues absolutely dread filling it in and do not do anything regularly till the final quarter of the year

Doctors also frequently mention a desire for a mobile app to streamline the recording process and allow for CPD credits to be recorded as they are obtained, rather than having to sit down at a PC 'a CPD phone app would be fantastic and really time-saving'. As many doctors, particularly in clinical practice, have intense workloads, a slow, time-consuming process for recording CPD credits does not fit easily into the workday.

\section{THEME 2: THE STRUCTURE OF PCS}

The second major theme to result from the analysis relates to perceived structural issues with the design of the PCS. This theme can be split into two main subthemes relating to both the content and quantitative requirements of the PCS.

\section{Issues with Content of CPD Categories}

The first subtheme describes doctors' dissatisfaction and confusion with the breakdown of the required CPD categories (internal, external, research and personal learning). Doctors perceive the division between these categories, as well as the eight domains of good professional practice, as being arbitrary and vague; 'The classifications are utterly artificial, arbitrary, confusing, overlapping and have little meaning in everyday practice'.

This idea that the real-world applicability of the CPD categories to everyday practice is limited was common throughout the data, particularly for doctors within more niche specialties 'The categories listed when explaining type of CPD are irrelevant to my specialty. The scheme is 
too generic. It is easy for people to comply with requirements without carrying out good quality CME (continuing medical education)'.

Another doctor notes the need for specific examples of activities within the CPD categories, and what they are worth in terms of CPD points, particularly for the internal category 'The categories are not intuitive... It would be useful to have examples... particularly in the internal category' and for those in non-clinical roles 'I'm currently enrolled in a PhD and found it hard to translate all the research work, writing publications and reading into CPD points.'

Following from the perception of the CPD categories as vague and confusing, was uncertainty about which category to assign any given CPD activity to, with one doctor noting that 'Categories often overlap' making it 'irritating to have to shift attendance/activity from one site to another to fill gaps'. This suggests that doctors often assign activities to CPD categories in order to fit the requirements, rather than due to any real meaningful distinction between them.

Indeed, some doctors argue instead that removing the barriers of the category labels could promote a more intuitive approach to CPD, which could encourage more meaningful engagement with CPD activities. 'Remove the draconian CPD categories. Doctors are chasing CPD points instead of learning, making CPD and CME a wasteful exercise in futility'.

\section{PCS is inflexible and unrealistic}

The second subtheme 'PCS is inflexible and unrealistic' highlights perceived issues with the structure of the PCS, in terms of both the quantitative category requirements (ie, no. of credits per category and Domain) and the yearly PCS requirements. Overall, this subtheme relates to doctors feeling restricted by the requirements of the scheme.

Relating to the specific requirements within each CPD category, doctors express concern that they are too rigid, and less achievable for certain specialties and areas of practice, particularly retired doctors, or those in-nonclinical roles: 'There is a need to provide for doctors engaged in other non-clinical roles especially where audits are concerned. Several of the domains are difficult to achieve unless hospital or team based.'

There is also a sense that some doctors feel that the PCS undervalues non-clinical skills with the larger credit requirements for internal and external CPD, with lower requirements for personal learning and research, even when the latter types of CPD may be more relevant to their own personal practice:

A huge part of my work $(60 \%+)$ involves work that is essentially me doing CPD but only a tiny part of that can be used as part of my CPD that is, personal learning... My actual real learning where I set out to improve my knowledge of an area I feel I don't know enough about is not actually recognised.
This suggests that doctors feel the PCS category credit requirements were designed for doctors who provide direct patient care, with doctors in different roles (academia, research, management, retirees, etc) forced to comply with requirements that may not be particularly relevant to their own work. Some doctors feel that the rigidity of the system was controlling, where 'doctors are being prescribed what they can learn'. This suggests a difficulty adapting and relating the CPD framework to their own scope of practice.

Participants highlight the fact that certain CPD categories (most notably internal) are difficult to participate in when the doctor has been out of clinical practice for an extended time (due to maternity leave, illness, etc). One female doctor describes her experience trying to meet her internal CPD requirements while on maternity leave:

I was advised to return to the institution that I was working in at the start of the CPD year to get my internal credits. I was told by that institution that for patient confidentiality reasons I could not attend any departmental meetings when I was not employed by the institution.

This quote illustrates the difficulty inherent in trying to participate in internal CPD when not currently working in clinical practice, with the onus on the individual doctor to organise the activities, while legal or administrative procedures in place in hospitals can complicate the process.

Indeed, the particular difficulty faced by female doctors on maternity leave led some study participants to believe that the system was biased against women with children, with one participant stating 'I feel that there is some gender bias in that there is no accommodation given to women on maternity leave in terms of CPD requirements. It is simply not possible to attend on site lectures, meetings etc while caring for an infant'.

It was suggested that alternate requirements could be fulfilled by women who were on leave 'the same CPD requirements should not be necessary or it there could be a different balance more in favour of self-directed learning rather than internal CPD for those on maternity leave'.

As well as the specific category requirements, study participants also tended to see the yearly PCS requirements as being restrictive, and out of step with the realities of life, with 'no allowance for unusual circumstances'. The structure of the scheme is seen by some as being unaccommodating for those who had to take a leave of absence, as well as failing to consider career transitions. As one doctor puts it, the scheme has a 'Lack of flexibility to reflect changing circumstances for example, from trainee to specialist, or support for maternity/ family leave'.

Another participant also notes the lack of accommodation for 'unusual' circumstances, while also suggesting that CPD points should be measured over 5 years rather than one "The scheme is too generic...the scheme is punitive for women on maternity leave or sick leave. There should be more protection for women/ill fellows/ 
participants. The original data which allowed the points to be cumulative over a 5-year cycle has not been borne out.'

Indeed, the lack of flexibility from restricting points totals to a 1-year time limit was also seen to influence which CPD activities doctors participated in, sometimes forgoing better, more relevant activities later in the year, in order to make sure they have enough CPD points by the deadline:

My specialty is histopathology. The main and arguably best international meeting is USCAP held in March in either USA or Canada. It can be difficult to know if one can attend until near the date of the meeting. Therefore, one tends to attend another international conference earlier in the May-April year to ensure one has enough external points. As a result, I find that if I have enough external points from a European meeting, the incentive to attend USCAP (attendant expense, time off work, often 2 flights) is diminished...

\section{THEME 3: BENEFITS OF PCS VERSUS CRITICISM OF FORMAL CPD}

Doctors also comment on formal CPD in general. Central to this theme is the perception that CPD is generally valued and seen as important, but for some, formal CPD is perceived as little more than a bureaucratic exercise, which creates more work for doctors; 'I have always been keen on my own professional development... Keeping up to date is my own professional interest and pride. Documenting those is a pure political interest.'

Doctors express understanding and positivity towards the need for continuous professional development, while questioning the need for a formal, credit-based system, noting that 'The PCS scheme relies too much on collecting points with insufficient evidence of learning or improvement' A number of doctors express scepticism that CPD in its current form has any benefit to either doctors or patients, and question whether there is sufficient evidence to support the practice; 'I have major reservations about the benefits of this whole process to the public'.

One doctor implies that the formal CPD process takes doctors away from patient care 'This controlling will not help improve professional practice. It rather stimulates doctors to do paperwork and detach from patient care.'

In addition to questioning the benefits of CPD participation, doctors also note that the added workload of CPD participation and recording only serves to exacerbate an already stressful career:

The burden of extra activity and work and demands on medical staff is enormous, only adding extra stress. I would advise not to increase demands on individuals

One doctor who had to engage in CPD during maternity leave describes the extreme stress the process caused her:
I feel that the system let me down and put a shadow of worry over my time at home with my baby that did not need to have been there. Also, it is very difficult to attend for CPD whilst breastfeeding a baby...The requirement to get CPD points during 'maternity leave' impaired my ability to optimally and safely nourish my baby.

\section{THEME 4: WORKPLACE CHALLENGES}

The fourth major theme refers to specific workplace-based challenges to participation in CPD, which relate strongly to the current difficulties being experienced in the Irish healthcare system. This theme also reflects the barriers frequently reported internationally in previous research; namely, lack of time and financial cost of participation. ${ }^{8}$

The first barrier identified within this theme is lack of time, both to participate in and record CPD activities. This lack of time was particularly pronounced for nonconsultant hospital doctors (NCHDs); as summarised by 'As with everything in medicine... time is an issue!'

In addition to noting the general lack of time given the demands of work, participants also specifically mention staff shortages and the related lack of protected study leave in contributing to the time pressure. Indeed, even when the perceptions of CPD are positive, time is seen as a barrier, with one doctor noting 'The professional competency scheme is an excellent opportunity to avail of external/personal learning opportunities. However, there is a lack of time as an NCHD to complete these tasks and this is often done in my leisure time to ensure my portfolio is up to date'.

Accordingly, a number of doctors express a desire for more online-based CPD, in order to allow time-stretched doctors to complete activities in their own time ('One should be able to get all CPD points via online courses as it is extremely difficult and causes huge work problems for NCHDs to take leave to attend courses').

Doctors also highlight the financial cost of participating in CPD activities, with numerous doctors noting the fact that CPD is just an added cost on top of other administrative financial burdens 'I am frustrated by the annual cost of enrolling in a Professional Competence Scheme. I have yet to encounter any satisfactory explanation as to why I must pay this additional cost on top of my licence retention fee with the Medical Council'.

\section{THEME 5: ACCESS ISSUES}

The final theme 'Access Issues' describes a number of specific factors which result in lack of access to high quality relevant $\mathrm{CPD}$ activities.

The first of these factors is the location of CPD activities, with doctors stating that the majority of existing activities are too Dublin-central, to the neglect of non-Dublin-based doctors by CPD providers; 'The CME programmes... are 
totally geared towards Dublin based doctors and makes it easier for them to attend and gain points more regularly'.

The second of these factors relates to technical and organisational difficulties as a barrier to accessing online CPD, on multiple fronts; slow internet on office computers, bad quality connections to webcasts, etc: 'There needs to be a method for ensuring that webcast presentations are technically of better quality, 1 am referring to the quality of both the visual and audio components of the webcast as all too frequently these are inadequate, at least in some locations, and so reducing their value.'

In addition to location and technical difficulties impeding access to CPD, some doctors note that there is a lack of relevant CPD activities themselves, particularly in niche specialty areas; 'Specialist practice makes accessing relevant CPD more challenging'. These findings indicate that doctors find it difficult to find relevant meaningful CPD activities in Ireland for smaller specialties, potentially due to smaller numbers of doctors within each specialty resulting in lower overall demand for niche courses. It is worth noting that that if people cannot access relevant CPD they still must fulfil their requirements, and therefore may have to devote significant time and effort into participating in non-relevant CPD. Indeed, one doctor working in a small specialty states, 'I find it a challenge to get meaningful professional development and so much of what I do is generic.'

\section{GENERAL DISCUSSION AND IMPLICATIONS}

While the vast majority of the 1408 doctors who responded to the larger RCPI survey-based study reported CPD 'a good idea' and that it improves patient care and safety, ${ }^{20}$ of the 434 doctors who responded to the open- ended question, a number of barriers and also suggested improvements were reported.

All five major themes (figure 1) related primarily to barriers and potential improvements to PCS participation, despite the open-ended question being phrased to allow comments on positive aspects and benefits to PCS. Taken together, the five themes outlined in this study give

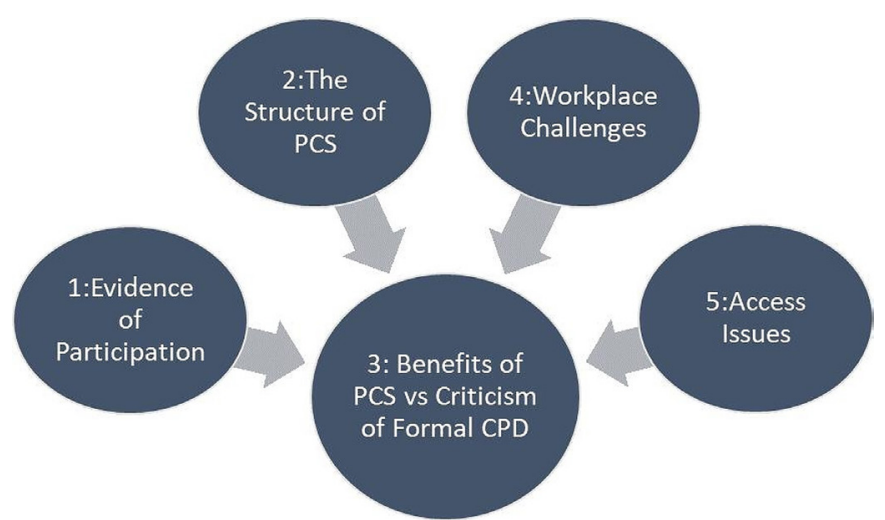

Figure 1 Five major themes relating to benefits, barriers and potential improvements to PCS. CPD, continuing professional development; PCS, Professional Competence Scheme. a wide-ranging, in-depth picture of the challenges faced by Irish doctors in 2018, which expand on 'established' barriers such as time constraints, to illustrate a series of complex, interacting factors.

The barriers outlined in themes 1 (evidence of participation), 2 (structural issues with PCS) and 4 (systematic issues, such as lack of time) likely interact with and contribute to the negative perceptions and beliefs that CPD activities are of limited benefit outlined in theme 3 , and result in a number of doctors feeling that mandatory CPD is irrelevant to practice and see it as an added stressor.

Theme 1 illustrates that it may be the case that doctors are attending relevant and useful CPD activities, but due to difficulties or delays in obtaining credits, they are unable to put them forward toward their end-ofyear totals. In addition, the lack of a simple method for recording CPD credits further impedes doctors' ability to meet the requirements of their PCS. The perceived difficulties with obtaining and recording evidence of participation in CPD activities represent a unique barrier to engagement with CPD that has not frequently emerged in previous research. Past studies have shown that doctors can view mandatory CPD as a bureaucratic hurdle, ${ }^{13}$ and it is conceivable that constant difficulties in gathering evidence of participation may add to this perception. In contrast to potentially more systemic issues impeding CPD engagement, such as a lack of time, the barriers outlined in theme 1 are relatively easily addressed. By streamlining the process and improving the ease at which doctors can obtain and submit evidence of their CPD activities, then a major barrier to successful engagement could be removed. Such changes have already begun to be implemented in various ways; an improved electronic platform and revised e-Portfolio which allows doctors to record their CPD and Audit activities in real-time was launched by RCPI in 2019.

The two subthemes of theme 2 emphasise a perceived lack of flexibility, little accounting for 'unusual circumstances' and retirement, and perceived bias in the PCS toward women on maternity leave. In general, theme 2 points toward criticism of a 'one-size-fits-all' approach to $\mathrm{CPD}$, both in terms of specific category and yearly requirements, and a desire for a system that is more open to individual medical specialties and which takes into account the realities of life. Any future revisions of the PCS system might take these requests for more flexibility both in terms of CPD categories and allowances for extenuating circumstances into account when setting CPD requirements.

Again, like theme 1, theme 2 represents a novel area in the context of existing research, with respondents highlighting a perceived inflexibility of the formal system and an inability to adapt the CPD framework to their individual scope of practice, a potential impediment to their meaningful engagement with relevant CPD. As mentioned, a large proportion of previous studies use forced-choice methods in which participants rate or 
endorse a range of predetermined potential barriers. Such methods necessarily miss out on more nuanced responses which can result from open-ended questions. Theme 2 then indicates that there may be more complex, less obvious barriers involved in formal CPD programmes other than the more immediate barriers of time pressure and financial cost.

The perception that formal CPD is of questionable benefit outlined in theme 3 is in line with previous research demonstrating that some doctors feel that CPD is merely a 'tick-box' exercise. ${ }^{912}$ As mentioned, previous studies have demonstrated that when people hold low task values toward a particular activity, they are less intrinsically motivated to engage with that activity. ${ }^{14}$ These negative perceptions of CPD as being an administrative burden without clear benefit could potentially impact doctors' motivations to participate in PCS, along with the additional perceptions that PCS participation can be a significant source of stress.

As previous research has consistently demonstrated CPD to be effective at improving doctor performance and patient outcomes, it is clear there is a gap between the observable impact of CPD and the beliefs of some doctors about its benefits. This may be partially accounted for by the kinds of CPD activities doctors in Ireland are participating in-for example, numerous review studies have shown interactive activities that use multiple methods over a longer period to be most effective at producing change than traditional didactic presentations. ${ }^{3}{ }^{4}$ For doctors working under conditions of staff shortage and financial restriction, access to relevant high quality and more intensive, longer-term CPD activities may be reduced. If doctors are engaging more often with less relevant and less-effective kinds of CPD activities, this could contribute to the perception that the costs of mandatory CPD outweigh its benefits.

It is likely that the other themes found in the data add to these perceptions of CPD as simply being an added burden. The perceived difficulties in obtaining and recording CPD credits put forward in theme 1 may contribute to frustration and negative attitudes, as even after completing CPD activities, an 'arduous' process must still be completed. Similarly, the perceived inflexibility of PCS described in theme 2 may contribute to negative perceptions of mandatory CPD, as those in niche-area and non-clinical practice may feel they are spending time and money completing CPD activities that they feel are not relevant or meaningful to their practice. Similarly, the perceived lack of accommodation for unusual circumstances such as sick leave may add to stress for doctors. Essentially, theme 3 outlines the perceived costs of participating in formal CPD; adding to an already overworked, highly stressed workforce with additional requirements and little perception of benefit.

Theme 4-relating to workplace challenges to PCS participation-is best understood in the context of the Irish healthcare system in 2018; in which hospital staff are overworked, with severe staff shortages and hospital overcrowding adding to the stress experienced by doctors. It appears that many doctors perceive CPD participation to be restricted by their current working environment. These barriers-time and cost-are the barriers most likely to be mentioned in previous research, especially in working environments experiencing staff shortages. ${ }^{6-8}$

The desire for more online-based CPD as a result of time restrictions is interesting, given the perceptions that CPD activities are of limited benefit outlined in theme 3. Previous research has shown online CPD to be the least effective in changing doctor behaviour or patient outcomes when compared with more interactive in-person activities, suggesting that doctors may favour CPD activities which have fewer tangible benefits to their practice. However, the reality is more complex; online CPD activities which involve discussion and have higher levels of interactivity and involve practice exercises have been found to improve knowledge and skills. ${ }^{15}$ A recent review of the efficacy of online forms of CPD spanning 88 studies found that online learning was of comparable effectiveness to face-to-face learning with regard improving knowledge, skills, and physician behaviour and patient outcomes, ${ }^{22}$ so it may be the case that the quality of online CPD activities is increasing. The authors note that regardless of the format of CPD, effective activities must be evidence based, interactive, involve some form of self-assessment activity, and multiple exposures to the learning material. ${ }^{22}$ Online CPD providers in future should aim to incorporate these factors, to maximise the efficacy of their activities while providing doctors with the benefits of cheaper and more accessible CPD.

It is possible that the access issues described in theme 5 only serve to compound the frustration felt by some doctors. Theme 5 highlights the sentiment that CPD activities focused mainly on urban centres may exclude many rural doctors. This perceived geographical disparity in service provision likely reflects the fact that Dublin, as the capital city, has the highest concentration of doctors in Ireland. This issue is also likely related to the barriers of time and cost outlined in theme 4-rural doctors have increased travel time and costs to get to CPD activities compared with doctors already situated in major cities. The experience of technical difficulties in accessing online CPD is important to note given the fact that many doctors offered location as a barrier; these doctors may be particularly impacted by being unable to engage in online CPD, which could otherwise help to make up lost CPD points due to being geographically distant from CPD activities. Similarly, the perceived lack of access to CPD activities for those who are not providing direct patient care and working in niche specialties reflects the beliefs throughout theme 2 that PCS schemes may be reflective of the 'average doctor' with less provisions for the outliers. By nature, formal CPD schemes need to cater for a large group of doctors and cannot account for every unique case and an adaptable CPD framework is provided, however the findings outlined in this study suggest that provision of a balance of learning activities is required to 
help ensure that niche groups-be it specialty, location or unusual circumstances-do not fall through the cracks. In addition, special allowances should be made for doctors who are ill, on maternity or compassionate leave.

As a result of circumstance, the nature of CPD activity in 2020 is radically different from that in 2018, when data were collected for this study. The COVID-19 pandemic has led to almost all CPD activities moving to a virtual learning format including a wide range of relevant interactive webinars which include national and international experts. Many formal 'traditional' activities, such as large, in-person international conferences have also moved online, offering global access to interactive learning activities with international experts along with a wide range of educational activities involving combinations of large and small group activities. This essentially eliminates many of the barriers identified by participants in this study; increasing accessibility for doctors in more remote locations, and reducing the time commitment and cost required to attend. The impact of this change on doctors' experiences and challenges engaging with PCS should be investigated in future research.

Finally, it is worth noting that it is encouraging that running throughout the dataset was a general consensus that CPD and lifelong learning are worthwhile and essential components of the practice of medicine. It is clear that in the future, that while learning from excellence is important, efforts must also be made to target the more negative perceptions of CPD, while also tackling the structural and financial barriers to CPD participation, such as lack of study leave. The barriers highlighted by doctors in this study are broadly in line with those found by another recent study based in Ireland. ${ }^{23}$

\section{Strengths and limitations}

The present study describes novel barriers to engagement with a formal system of mandatory CPD, with the qualitative nature of the study allowing for a more in-depth examination of the issues outlined by participants (eg, the structure of PCS) which have not been examined in previous work, at a level of detail that is often missed in quantitative studies. This study is limited by its use of a single open-ended anonymous question, with no opportunity for follow-up questions. However, future research is planned to expand on the findings of this study in more depth. In addition, it must be noted that a majority of participants did not respond to this optional question, so it may be the case that responses disproportionately represented those with more complaints about formal CPD. However, this group-those who perceive and experience significant barriers to PCS participation-are the group who are least likely to meet their CPD requirements, and therefore, their needs must be identified in order to design interventions aimed at improving their performance. Additionally, when examining the general attitudes towards PCS among respondents versus nonrespondents to the qualitative question, the proportion of participants who agreed that PCS was a good idea overall was the same, $80 \%$ agreement for both groups, suggesting no particular prejudice against the idea of formal CPD among the respondents in this paper.

Future research should focus in on the individual needs of specific vulnerable groups of doctors, in order to tailor interventions more precisely, and maximise performance across the board going forward. Future research should also examine whether the shift towards virtual learning as a consequence of the COVID-19 pandemic has impacted on doctors' experiences of engaging with PCS.

\section{Twitter Lucia Prihodova @LuciaPrihodova}

Contributors All authors (HRH, LP, TR, DD, HH and AO'S) were involved in the conception, design and planning of the measures used in the study, and in the recruitment and implementation of the study. Analyses were conducted by HRH and reviewed by LP. HRH drafted the manuscript and all authors were involved in revising it for publication. HRH is responsible for the overall content of the article as guarantor.

Funding The authors have not declared a specific grant for this research from any funding agency in the public, commercial or not-for-profit sectors.

Competing interests All authors are employed by the Royal College of Physicians of Ireland (RCPI), which manages a number of Professional Competence Schemes (PCS) in Ireland. PCS is funded by doctors' registration fees.

Patient and public involvement Patients and/or the public were not involved in the design, or conduct, or reporting, or dissemination plans of this research.

Patient consent for publication Not applicable.

Ethics approval Ethical approval for this study was granted by the RCPI Research Ethics Committee.

Provenance and peer review Not commissioned; externally peer reviewed.

Data availability statement Data are available on reasonable request. As per the ethical approval, the data will not be shared outside of the participating research institutions. Any sharing of the data beyond the group will be subject to review by the host institution (Royal College of Physicians of Ireland) and to independent research ethics application. Any queries on how to access the dataset should be directed to research@rcpi.ie.

Open access This is an open access article distributed in accordance with the Creative Commons Attribution Non Commercial (CC BY-NC 4.0) license, which permits others to distribute, remix, adapt, build upon this work non-commercially, and license their derivative works on different terms, provided the original work is properly cited, appropriate credit is given, any changes made indicated, and the use is non-commercial. See: http://creativecommons.org/licenses/by-nc/4.0/.

ORCID iD

Holly Rose Hanlon http://orcid.org/0000-0002-8311-5377

\section{REFERENCES}

1 Aparicio A. Continuing professional development for doctors, ceritifcation, licensure and quality improvement. A model to follow? Educación Médica 2015;16:50-6.

2 Solé M, Panteli D, Risso-Gill I, et al. How do medical doctors in the European Union demonstrate that they continue to meet criteria for registration and licencing? Clin Med 2014;14:633-9.

3 Bloom BS. Effects of continuing medical education on improving physician clinical care and patient health: a review of systematic reviews. Int J Technol Assess Health Care 2005;21:380-5.

4 Cervero RM, Gaines JK. The impact of CME on physician performance and patient health outcomes: an updated synthesis of systematic reviews. J Contin Educ Health Prof 2015;35:131-8.

5 Brigley S, Johnson C, Bird J, et al. Hospital doctors' views of their $\mathrm{CPD}$ and its relationship to learning in the organization. Med Teach 2006;28:379-81.

6 Maher B, Faruqui A, Horgan M, et al. Continuing professional development and Irish Hospital doctors: a survey of current use and future needs. Clin Med 2017;17:307-15.

7 Etherton-Beer C, Katz B, Naganathan V, et al. Survey of Australasian geriatricians' satisfaction with, and preferences for, continuing professional development. Intern Med J 2016;46:805-11. 
8 Weiland TJ, Dent AW, Paltridge D. Australasian emergency physicians: a learning and educational needs analysis. Part three: participation by FACEM in available CPD: what do they do and do they like it? Emerg Med Australas 2008;20:156-63.

9 Dent AW, Weiland TJ, Paltridge D. Australasian emergency physicians: a learning and educational needs analysis. Part five: barriers to CPD experienced by FACEM, and attitudes to the ACEM mops programme. Emerg Med Australas 2008;20:339-46.

10 Ikenwilo D, Skåtun D. Perceived need and barriers to continuing professional development among doctors. Health Policy 2014;117:195-202.

11 Byrne BJ, Frintner MP, Abraham HN, et al. Attitudes and experiences of early and Midcareer pediatricians with the maintenance of certification process. Acad Pediatr 2017;17:487-96.

12 Macdougall C, Epstein M, Highet L. Continuing professional development: putting the learner back at the centre. Arch Dis Child Educ Pract Ed 2017;102:249-53.

13 Archer J, Nunn S, Regan de Bere S, de Bere SR. The McDonaldization of appraisal? doctors' views of the early impacts of medical revalidation in the United Kingdom. Health Policy 2017;121:994-1000.

14 Dietrich J, Viljaranta J, Moeller J, et al. Situational expectancies and task values: Associations with students' effort. Learning and Instruction 2017;47:53-64.

15 Cook DA, Levinson AJ, Garside S, et al. Internet-based learning in the health professions: a meta-analysis. JAMA 2008;300:1181-96.
16 Medical Council. Available: https://www.medicalcouncil.ie/ExistingRegistrants-/Professional-Competence/Professional-CompetenceSchemes/ [Accessed 26 Feb 19].

17 Royal College of Physicians of Ireland (RCPI). CPD explained. Available: https://www.rcpi.ie/professional-competence/ information-for-enrolled-doctors/cpd-explained/ [Accessed 17 Feb 2020].

18 Lindsay E, Wooltorton E, Hendry P, et al. Family physicians continuing professional development activities: current practices and potential for new options. Can Med Educ J 2016;7:e38-46.

19 Thomas S, Burke S, Barry S. The Irish health-care system and austerity: sharing the pain. Lancet 2014;383:1545-6.

20 Hanlon HR, Prihodova L, Hoey H, et al. Attitudes, perceived benefits, and experiences of engagement with professional competence schemes for doctors in Ireland: findings from a national survey. $J$ Contin Educ Health Prof 2021;41:176-184.

21 Braun V, Clarke V. Using thematic analysis in psychology. Qual Res Psychol 2006;3:77-101.

22 Ngenzi JL, Scott RE, Mars M. Information and communication technology to enhance continuing professional development (CPD) and continuing medical education (CME) for Rwanda: a scoping review of reviews. BMC Med Educ 2021;21:1-8.

23 Galvin E, Wiese A, Dahly D, et al. Maintenance of professional competence in Ireland: a national survey of doctors' attitudes and experiences. BMJ Open 2020;10:e042183. 